\title{
Modelagem ElÉtrica dos Tubos de RAIOS-X \\ UTILIZADOS EM SISTEMAS DE FLUOROSCOPIA COM O \\ e-Xactal \\ ObJetivo de Minimizar a Radiação Transmitida aO \\ PACIENTE
}

ISSN: 1984-3151

\author{
Electrical Modeling of X-Ray Tubes uSed in Fluoroscopy \\ Systems WITH THE OBJective Of Minimizing RAdiation \\ Transmitted to Patients
}

\begin{abstract}
Raghunatha Faria Duarte ${ }^{1}$; Bruno Andrade de Oliveira ${ }^{2}$; Euzebio de Souza ${ }^{3}$
1 Graduado em Engenharia Elétrica. Centro Universitário de Belo Horizonte - UniBH. Belo Horizonte, MG. raghunathafaria@gmail.com.

2 Graduado em Engenharia Elétrica. Centro Universitário de Belo Horizonte - UniBH. Belo Horizonte, MG. brunodoliver90@hotmail.com.

3 Mestre em Engenharia Elétrica. UFMG, 2013 Professor do UniBH. Belo Horizonte, MG euzebio.souza@prof.unibh.br.
\end{abstract}

Recebido em: 28/02/2017 - Aprovado em: 12/05/2017 - Disponibilizado em: 31/05/2017

RESUMO: A finalidade deste artigo foi demonstrar os processos e parâmetros e que são necessários para o funcionamento do tudo de raios- $X$ na utilização em técnica de fluoroscopia, abordou-se a parte construtiva do Gerador de alta tensão e do tubo de raios- $X$ e a forma de como ocorre a liberação dos raios- $X$ que incide ao paciente durante o exame, a condição de como a corrente e a diferença de potencial geram os raios- $X$ que incide ao paciente durante o procedimento e o que uma exposição desnecessária a tais raios pode ser prejudicial em quantidades que não agregam imagem ao exame, desta forma criou-se uma proposta de modelagem elétrica de um tubo de raios- $X$ em que abordou uma alternativa para minimizar a radiação que atravessa ao paciente sem a geração de imagem útil.

PALAVRAS-CHAVE: Modelagem. Tubo de raios-X. Minimizar.

ABSTRACT: The purpose of this article was to demonstrate the processes and parameters that are necessary for the operation of all $X$-rays in the use in fluoroscopy technique, the constructive part of the High Voltage Generator and the $X$-ray tube and the How the $X$-ray release occurs to the patient during the examination, the condition of how the current and potential difference generates the $X$-rays incident to the patient during the procedure and what unnecessary exposure to such rays May be detrimental in amounts that do not add an image to the examination. In this way, a proposal was made for the electric modeling of an X-ray tube, in which it addressed an alternative to minimize the radiation that passes through the patient without generating useful images.

KEYWORDS: Modeling. X-ray tube. Minimize. 


\section{INTRODUÇÃo}

Neste artigo será apresentada uma modelagem de circuito elétrico, em que seu comportamento se assemelha ao de um tubo de raios- $\mathrm{X}$, que é utilizado em equipamentos que possibilitam a realização de exames de fluoroscopia.

Nesse sentido será abordado de forma abrangente o funcionamento de gerador de alta tensão utilizado para alimentar o tubo de raios-X. Tais parâmetros fornecidos que viabilizam o uso do tubo de raios-X em procedimentos não evasivos, consistindo estes na corrente de filamento, em que ocorre a disponibilização de elétrons, a diferença de potencial entre as suas extremidades que direcionam os elétrons que são liberados para colidirem com o anodo rotatório e a tensão necessário para que o anodo gire.

No fornecimento de corrente para o filamento e tensão entre as extremidades do tubo a radiação é liberada, proporcional às intensidades das alimentações. Deve existir uma variação de radiação emitida devido à diferença de massa corpórea que muda de acordo com a região do exame.

Com base nas informações e parâmetros do funcionamento do tubo de raios- $X$, será apresentada uma modelagem, que o seu resultado propõe uma forma de se atenuar a emissão de radiação que incide ao paciente e que não produz imagem útil durante 0 exame de fluoroscopia.

\section{FLUOROSCOPIA}

Em novembro de 1895, o professor Wilhelm Conrad Roentgen encontrou um lugar permanente na medicina e em outras atividades com a descoberta dos raios- $X$. As imagens radiográficas podiam ser obtidas tanto por exposição de chapas fotográficas (para produzir imagens fixas) como por meio de um objeto entre o tubo de raios- $\mathrm{X}$ e uma tela fluorescente (DUFFIN; HAYTER, 2000).

$\mathrm{Na}$ sequência de sua descoberta o raio invisível foi experimentado obstinadamente para testar suas propriedades. Ele logo observou que os objetos sólidos colocados entre o tubo e a tela fluorescente atenuavam ou bloqueavam dependendo de sua densidade e ou estrutura (LINTON, 1995).

Em janeiro de 1896 o cientista italiano Enrico Salvioni inventou a Fluoroscopia, cujo dispositivo de mão chamado fluoroscope montado consistia em uma caixa oblonga sendo que uma extremidade da caixa se encaixa firmemente entre os olhos e a outra extremidade possuía uma tela fluorescente. Tal conceito é básico e é usado nos dias atuais (LINTON, 1995).

O físico italiano Enrico Salvioni inventou o primeiro fluoroscópio, porém foi 0 inventor e cientista americano Thomas Alva Edison que desde a sua descoberta se interessou pelos raios- $\mathrm{X}$, e com isso aperfeiçoou e o comercializou o primeiro fluoroscópio portátil em 1896 que consistia em uma tela fluorescente sensível aos raios- $X$ que mostrava a imagem sem a necessidade de radiografar fotograficamente. O vitescope Fluoroscopy foi comercializado e tornou se muito popular na época, principalmente, no campo da medicina, permitindo a observação de imagens internas do corpo humano em movimento (LINTON, 1995).

Um dos primeiros médicos e um dos poucos a ser intimamente familiarizado e a se especializarem em raios-X foi o Dr. Francis Henry Williams, de Boston, que foi fundamental para o uso inicial para os equipamentos de raios-X para o diagnóstico médico, incluindo a utilização de Fluoroscopia para o estudo de vasos sanguíneos que mais tarde ficou conhecido como angiografia (LINTON, 1995).

Pode-se entender a fluoroscopia como uma técnica muito utilizada na medicina atual que permite que o 
médico avalie estruturas internas do corpo humano como sistema digestivo, respiratório, estrutura esquelética entre outros sem a necessidade de uma intervenção no corpo humano. Ainda sendo usada com técnicas de ultrassom, radiografia, tomografia ou até em ressonância nuclear magnética, além disso, a Fluoroscopia tem um papel fundamental para a realização do cateterismo, que consiste no diagnóstico de obstruções nas artérias e a Fluoroscopia possibilita o visualizar o exame em tempo real através de monitores (SILVA, 2011).

Esse exame consiste no diagnóstico dos movimentos das estruturas internas através da realização de imagens de raios- $X$ em tempo real por meio de obtenção os fótons que incidem em um sistema de aquisição de imagem através de monitores de alta resolução para visualização (PARIZOTI, 2008).

A Figura 1 mostra um exame em Fluoroscopia no momento da inserção do catéter e liberação do contraste venoso radiopaco.

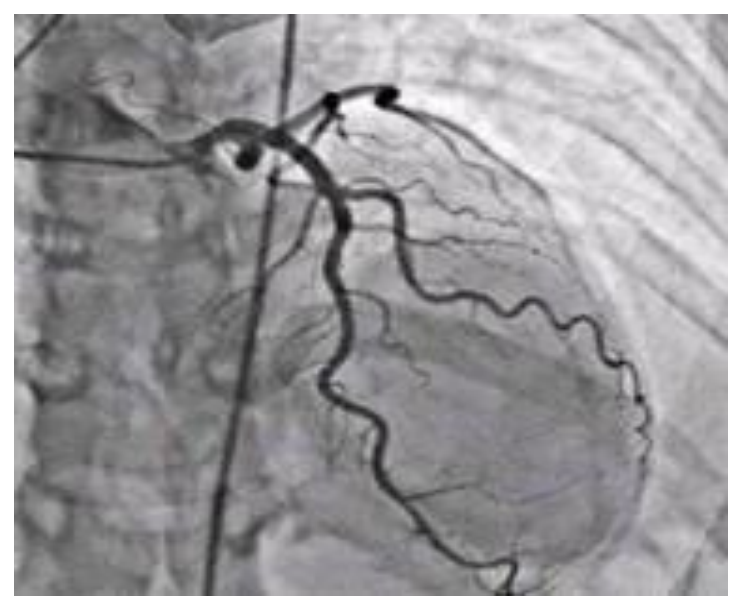

Figura 1 - Imagem do momento que o contraste é inserido na corrente sanguínea

Fonte - WOMENS HEALTH ADVICE, 2013.

\section{Gerador de Alta Tensão}

O gerador de alta tensão em um exame de Fluoroscopia tem a função de fornecer os parâmetros necessários para o tubo de raios- $X$, que através de um sistema de controle realizado durante o exame, 0 mesmo determina o quanto de tensão, corrente e velocidade de giro do motor do anodo, de acordo com a massa do paciente. Tomando como base o gerador de alta tensão que compõe um sistema de angiografia, de acordo com seu manual de operação, a faixa que um exame de Fluoroscopia trabalha com os seguintes parâmetros, apresentados na Tabela 1.

Tabela 1

Parâmetros para operação usados em exames de Fluoroscopia

\begin{tabular}{l|c}
\hline \multicolumn{2}{c}{ Parâmentos de operação } \\
\hline kV mínimo & $50 \mathrm{kV}$ \\
kV máximo & $120 \mathrm{kV}$ \\
Corrente mínima & $50 \mathrm{~mA}$ \\
Corrente máxima & $80 \mathrm{~mA}$ \\
Largura de pulso mínimo & $2 \mathrm{~ms}$ \\
Largura de pulso máxima & $12 \mathrm{~ms}$ \\
\hline
\end{tabular}

Fonte - Próprio autor.

O sistema de controle do gerador determina o quanto de tensão e corrente será enviado ao tubo para que o médico obtenha uma imagem satisfatória, bem como o tempo que o paciente ficará exposto durante o procedimento.

A Figura 2 demostra a ligação física do gerador com o tubo de raios- $X$. 


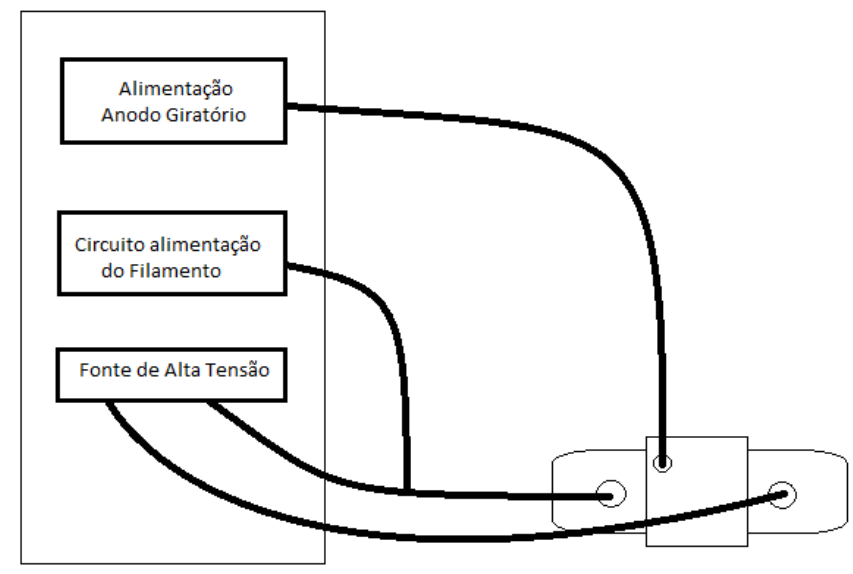

Gerador De Alta Tensão

Tubo de Raios- $X$

Figura 2 - Ligação do gerador de alta tensão no tubo de raios- $x$

Fonte - Próprio autor.

\subsection{Circuito Gerador de Alta Tensão}

A função do circuito é gerar a alta tensão capaz de criar a diferença de potencial que acelera os elétrons livres presentes dentro do tubo de raios- $X$, sua tensão e gerada de uma rede de $380 \mathrm{Vac}$, no qual o gerador é alimentado, essa tensão alternada passa por um retificador trifásico que a transforma em uma tensão retificada, passando por uma fonte chaveada ela é modulada através de um circuito PWM (Pulse Width Modulation).

Em seguida percorre um filtro LC com o intuito de retirar as componentes continua presente no circuito, essa tensão pulsada entra no primário de um transformador aumentador, depois em um dobrador de tensão e por fim é novamente retificada para alimentar o tubo de raios-X. Como demonstrado na Tabela 1 os parâmentos de operação do gerador, em exame de Fluoroscopia o gerador pode fornecer para o tubo de raios-X uma tensão entre $50 \mathrm{kV}$ a $120 \mathrm{kV}$ de acordo com o tipo de paciente e a parte do corpo a ser analisada.

O diagrama em blocos do circuito para geração de alta tensão está exemplificado na Figura 3.

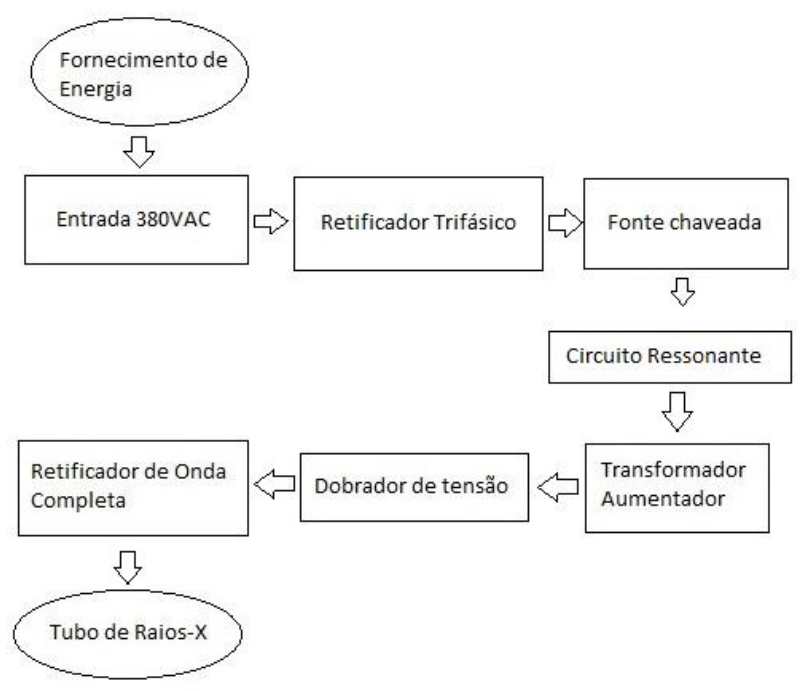

Figura 3 - Diagrama em bloco do circuito da fonte de alta tensão.

Fonte - Próprio autor.

\subsection{Filamento}

O circuito do filamento é responsável por gerar uma corrente necessária para percorrer o filamento de tungstênio e liberar os elétrons dentro do tubo de raios-X. Um modelo de tubo de raios- $X$ escolhido para esse estudo, trabalha com uma corrente de até $5.4 \mathrm{~A}$ em operação.

A Figura 4 mostra que de acordo com 0 valor de tensão $(\mathrm{kV})$, qual corrente é necessária ao circuito para percorrer o filamento de tungstênio e gerar os elétrons que irão colidir com o anodo girante gerando os fótons. 


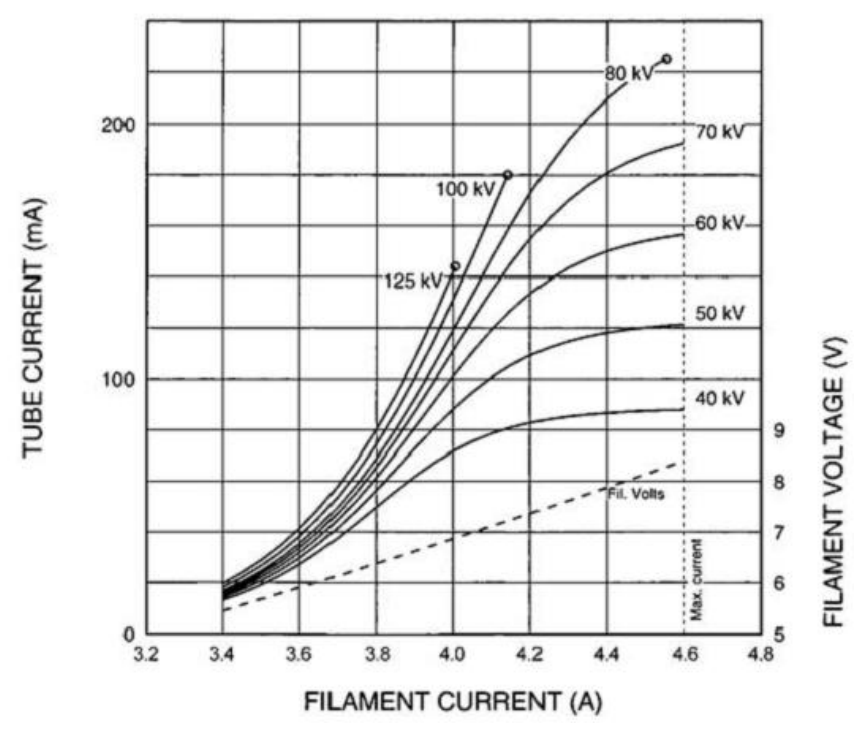

Figura 4 - Corrente no filamento $X$ tensão no tubo de raios- $X$

Fonte - VARIAN MEDICAL SYSTEMS, 2016.

O circuito do filamento tem sua alimentação proveniente de uma fonte de $48 \mathrm{Vcc}, 200 \mathrm{~W}$. Na sua saída, essa corrente retificada que sai da fonte vai passar por um circuito de ponte $\mathrm{H}$ que irá chaveá-la por meio de uma modulação PWM (Pulse Width Modulation), na qual a corrente passará a ser pulsada e então enviada para um transformador isolador e conectará no tubo de raios- $X$ alimentando o filamento de tungstênio com a corrente necessária.

No diagrama de blocos da Figura 5 será demonstrada a função dos componentes presentes no circuito do filamento.

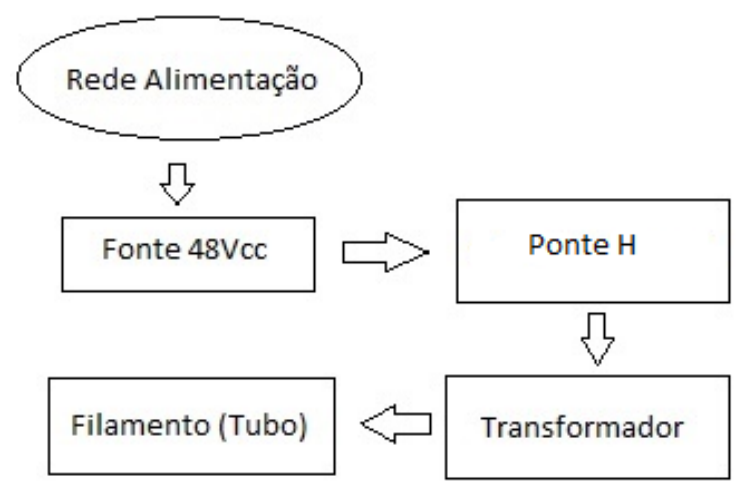

Figura 5 - Diagrama de bloco para o circuito do filamento.

Fonte - Próprio autor.

\section{Tubo de RaIOS-X}

Os tubos de raios- $X$ também podem ser chamados de tubos de Coolidge, que são nomeados dessa forma devido ao modelo de desenho original de W.C. Cooligde, que foi apresentado em 1913. O equipamento básico para a geração de raios- $X$ (WHITE; PHAROAH, 2004) .

O tubo de raios-X é composto de um anodo e um catodo, cujo o catodo serve como fonte ou diferença de potencial para o fluxo de elétrons que se deslocam para o anodo. O catodo e o anodo encontram-se dentro de um tubo de vidro fechado a vácuo. Quando os elétrons provenientes do catodo colidem com o anteparo situado no anodo ocorre à emissão de raios$X$ (WHITE; PHAROAH, 2004).

$\mathrm{Na}$ Figura 6 é descrito o nome dos principais componentes de um tubo de raios- $X$.

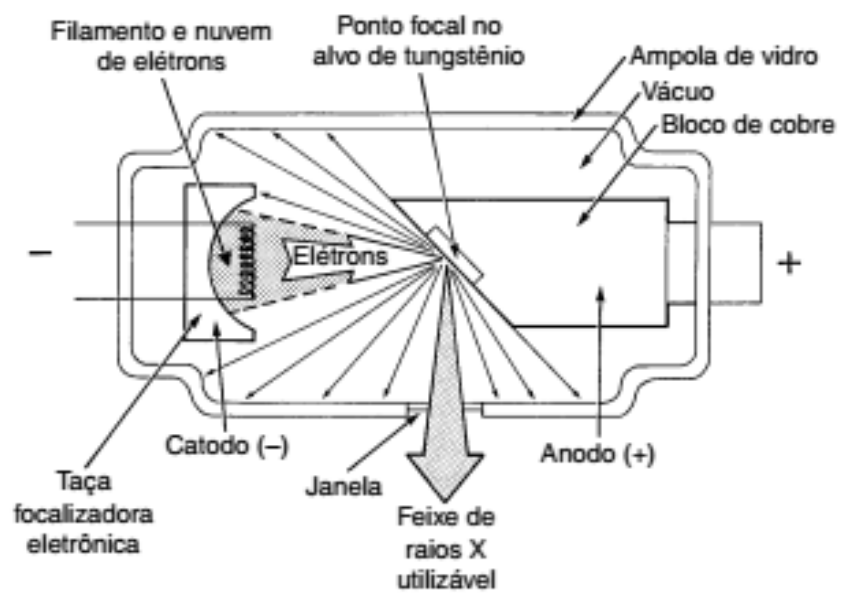

Figura 6 - Tubo de raios- $X$ com o nome dos principais componentes

Fonte - WHITE; PHAROAH, 2004, p. 07.

O tubo de raios-x especificado no presente estudo é o modelo G-1582BI, (VAREX IMAGING, 2017) do fabricante Varian Medical system, como demostrada a Figura 7. 


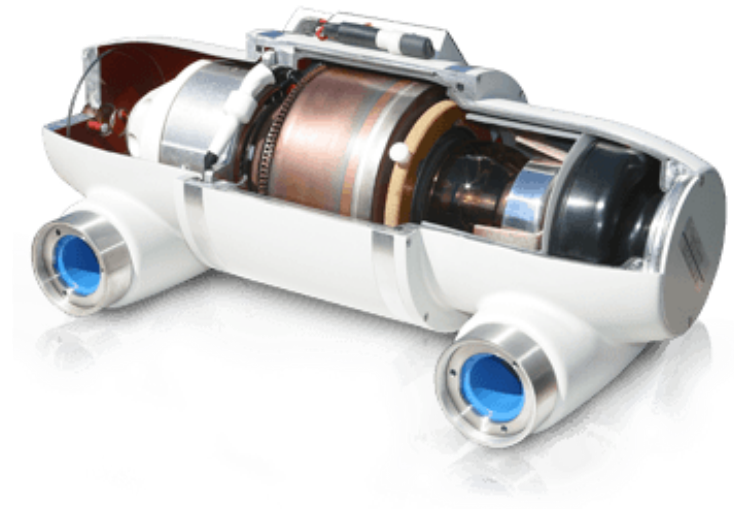

Figura 7 - Modelo estrutural do tubo de raios- $x$ varian

Fonte - VAREX IMAGING, 2016.

\subsection{Corrente no Tubo de RaIos-X}

Para o funcionamento do tubo de raios-X é necessário o aquecimento do filamento que possui uma forma de espiral, feito de tungstênio e tamanho de cerca de 2 $\mathrm{mm}$ de diâmetro e $1 \mathrm{~cm}$ de comprimento. Os elétrons provem do filamento (WHITE; PHAROAH, 2004).

A Figura 8 mostra que o filamento possui dois tipos de circuito, que estão ligados em conjunto a uma extremidade formando um conector comum, e outra extremidade dos filamentos estão ligados em transformadores que alimentam os mesmos através de um cabo de alta tensão que possui três condutores para controle independente de cada um dos focos do filamento. Eles se conectam a fontes de baixa tensão e o filamento é aquecido até que o fluxo de corrente proveniente da fonte emita elétrons (WHITE; PHAROAH, 2004).

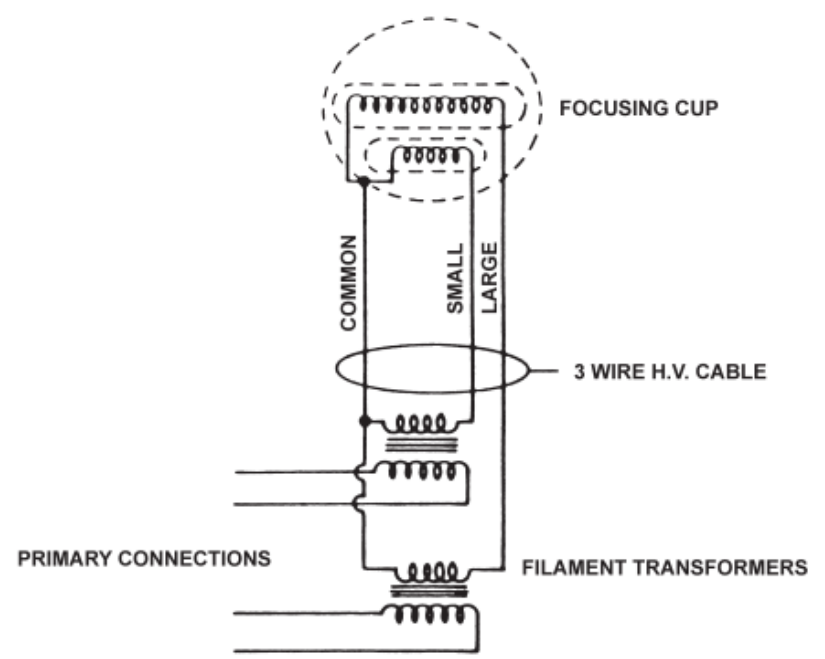

Figura 8 - Esquema do circuito do filamento exemplificado na parte interna do tubo de raios- $x$ Fonte - VARIAN MEDICAL SYSTEMS, 2014.

No filamento encontra-se a taça focalizadora que é um refletor côncavo carregado negativamente que direciona os elétrons livres para um feixe estreito onde possui uma área retangular do anodo, chamada de área focal, os elétrons livres caminham para essa área porque são repelidos pelo catodo que se encontra negativamente carregados e são atraídos pelo anodo positivamente carregado (WHITE; PHAROAH, 2004).

Com uma incidência maior de feixes de elétrons pode ser usadas correntes que variam na faixa de 100 a $500 \mathrm{~mA}$, ou seja, de 10 a 50 vezes mais do que com um anodo estático. Dentro de um tubo com anodo giratório, o rotor gira aproximadamente 10000 rotações por minuto e seu uso é mais frequente em aparelhos de raios- $X$ que requerem uma saída com mais radiação (WHITE; PHAROAH, 2004).

\subsection{ANODO}

O anodo é constituído por uma placa de tungstênio anexado a um bloco de cobre que auxilia na dissipação do calor gerado. Considerando que o cobre é um bom condutor térmico (para anodo estático) e para um anodo rotatório o tungstênio é na forma de 
um disco chanfrado que gira no momento que o tubo está em operação (WHITE; PHAROAH, 2004).

Os elétrons são convertidos em fótons de raios- $X$ ao colidirem com o disco do anodo. A Figura 9 representa um tubo de catodo rotatório com a incidência dos elétrons e o feixe produzido (WHITE; PHAROAH, 2004).

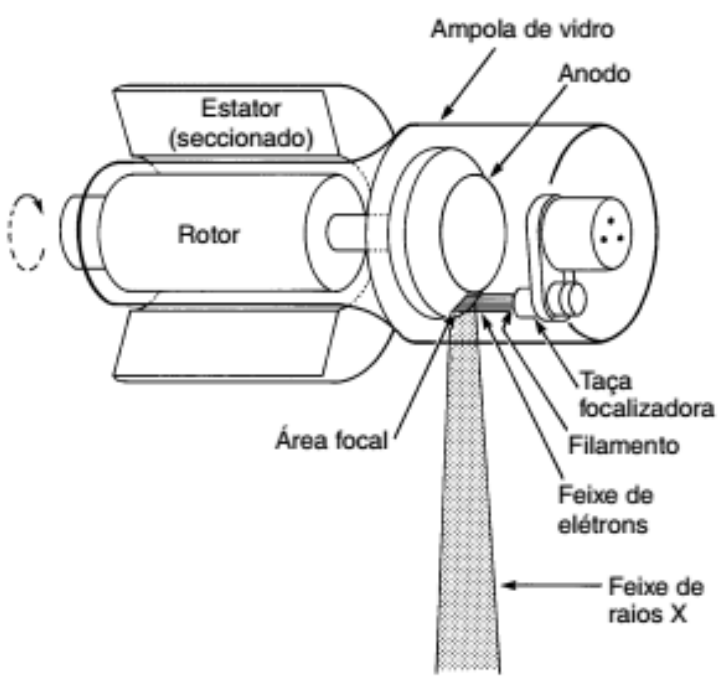

Figura 9 - Anodo rotatório de um tubo de raios- $x$ Fonte - WHITE; PHAROAH, 2004, p. 09.

De acordo com manual de operação do tubo de raiosX modelo G-1582BI, rotor do anodo pode operar em velocidades como demonstrados na Tabela 2.

Tabela 2

Frequência associada a velocidade por minuto

\begin{tabular}{c|c}
\hline \multicolumn{2}{c}{ Frequência no estator RPM } \\
\hline $50 \mathrm{~Hz}$ & $2800-3000$ \\
\hline $60 \mathrm{~Hz}$ & $3400-3600$ \\
\hline $150 \mathrm{~Hz}$ & $8500-9000$ \\
\hline $180 \mathrm{~Hz}$ & $9500-10,800$ \\
\hline
\end{tabular}

Fonte - VARIAN MEDICAL SYSTEMS, 2014.
De acordo com a Tabela 2 pode-se observar que quanto maior a frequência de operação no motor do anodo, maior será a velocidade de giro do rotor, essa dedução pode ser comprovada usando a Equação 1.

$$
n=\frac{120}{p} x F
$$

Em que:

O número 120 representa uma constante da fórmula

$n$ : Velocidade síncrona em RPM

$p$ : número de polos.

$F$ : Frequência em Hertz.

Por exemplo, se usar o uma frequência de alimentação do anodo a $180 \mathrm{~Hz}$, na Equação 2 tem-se o resultado da sua velocidade de operação como demostrado abaixo:

$$
n=\frac{120}{2} x 180
$$

O que demostra que quanto maior sua frequência de operação maior sua velocidade de giro.

\subsection{Tensão no Tubo de Raios-X}

Para ocorrer a emissão de raios- $\mathrm{X}$ o tubo deve ser alimentado com tensão retificada. Essa tensão controla o fluxo de elétrons entre 0 anodo e o catodo já que ela que é responsável por acelerar os elétrons dentro da ampola do tubo de raios-X (WHITE; PHAROAH, 2004).

Para cada tipo de equipamento e técnicas empregados no radiagnóstico, há um tipo de tensão de operação e possui uma faixa especifica que se trabalha, conforme Tabela 3. 
Tabela 3

Faixa de tensão $(\mathrm{kV})$ para cada tipo de exame

\begin{tabular}{c|c}
\hline Tipos de exames & Tensão Aplicada $(\mathrm{kV})$ \\
\hline Radiologia Convencional & 20 a 150 \\
Fluoroscopia & 50 a 120 \\
Tomografia & 20 a 150 \\
Mamografia & 22 a 40
\end{tabular}

Fonte - MIRANDA, 2009.

\section{TEMPO DE EXPOSIÇÃO}

O termo radiação ionizante descreve que o transporte de energia pode ser feito de duas formas, tanto pela forma de ondas eletromagnéticas como por partículas subatômicas (FLÔR ;KIRCHHOF, 2006).

Durante um exame radiológico, a geração de imagens ocorre devido à radiação na qual atravessa o corpo do paciente, podendo haver algum tipo de dano biológico ao mesmo (MADELON ; SILVA, 2015).

De acordo com a Ministério da Saúde (1998), a portaria 453 da secretaria da vigilância sanitária descreve que a duração deve ser a menor possível com a produção de uma boa imagem utilizando um receptor de imagem o mais sensível onde possa fornecer um nível de qualidade maior e mais detalhado.

Segundo White e Pharoah (2004), o tempo de exposição é o período ao qual o paciente fica exposto à radiação ionizante e quando este é aumentado duas vezes o número de fótons consequentemente é duplicado em todas as faixas do expecto, porém não se altera a gama dos fótons, logo como mostra a Figura 10 , se mudar o tempo de exposição irá controlar a quantidade da exposição ou só número dos fótons gerados.

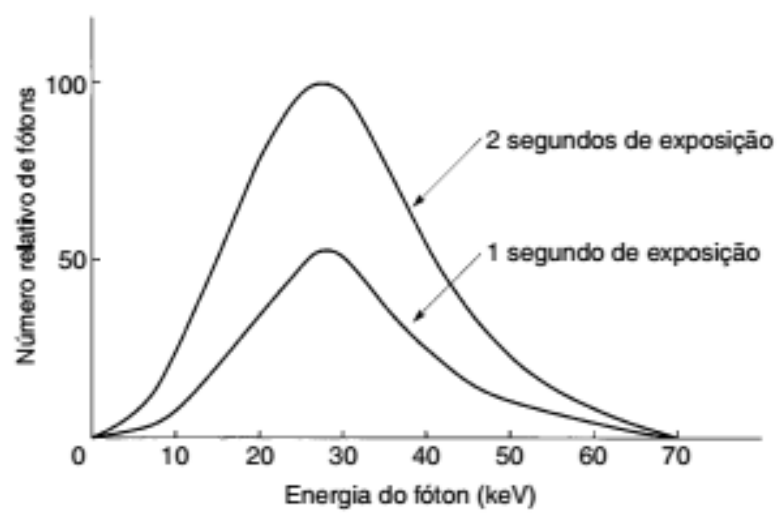

Figura 10 - Espectro de energia de fótons com o aumento do tempo de exposição Fonte - WHITE; PHAROAH, 2004, p.14.

\section{Modelagem do Circuito Elétrico Equivalente do Tubo de RaIOS-X}

Segundo Corrêa e Aguirre (2004), caixa cinza é uma categoria de técnica que se coloca entre a modelagem pela física ou natureza do processo (caixa branca) e a identificação (caixa preta). É através de informação auxiliar que essa categoria de modelagem se distingue, informação essa que não é possível ser localizar no conjunto que se utiliza durante a identificação (caixa preta).

Dessa maneira, a caixa cinza neste artigo pode ser entendida como uma modelagem, que através de parâmetros e estudos realizados no sistema do gerador e no tubo de raios- $X$, sendo proposto um circuito equivalente de forma a apresentar simulações para um funcionamento próximo do real.

Realizando as etapas mostradas no Fluxograma de Corrêa e Aguirre (2004), Figura 11, será possível criar uma caixa cinza para o circuito proposto:

Conforme o fluxograma da Figura 11, foi apresentado um passo a passo para a construção da caixa cinza para o estudo desse artigo: 
1. Selecionar a estrutura do modelo: Para o desenvolvimento do modelo utilizaram-se componentes passivos que serão capazes de nos fornece ao fim da montagem do circuito a forma de onda desejada.

2. Estimar os parâmetros na estrutura selecionada: Como parâmetro para essa etapa adota-se a forma de onda apresentada na Figura 12, pois será essa onda no qual foi utilizado para desenvolver o modelo.

3. Especificar alternativas na estrutura: Para atender ao modelo desenvolvido simula-se a redução da onda de descarga, usando componentes passivos.

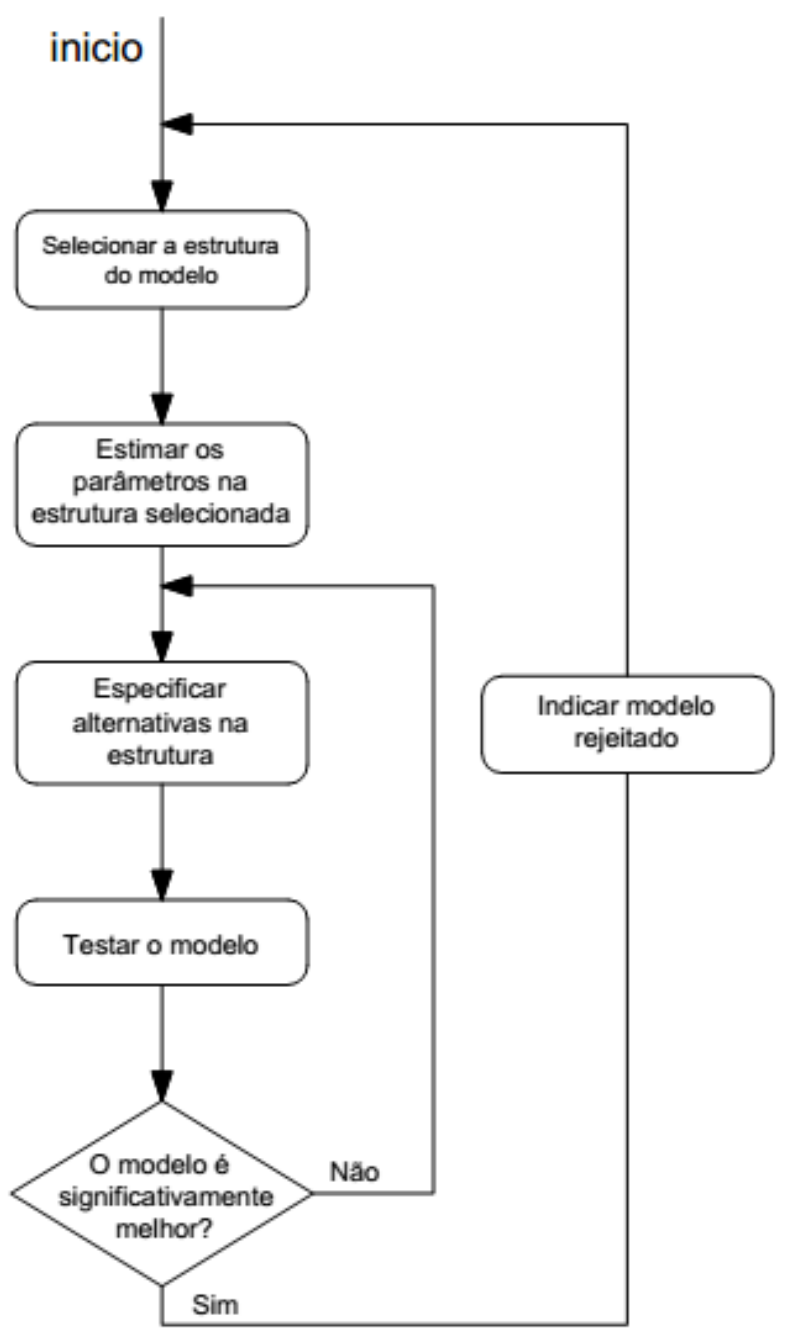

Figura 11 - Fluxograma ilustrativo do procedimento interativo de refinamento de estrutura (Bohlin e Graebe, 1995).

Fonte - CORRÊA; AGUIRRE, 2004.
4. Testar o modelo: Montado o circuito, conforme mostra a Figura 15, realizaram-se os testes do modelo criado e sua forma de onda na saída está representada na Figura 16.

5. Indicar modelo rejeitado: Em caso de sucesso no desenvolvimento da caixa cinza, os modelos anteriormente testados que não tiveram exito vederão ser apresentados.

\section{Metodologia}

Na Figura 12, a imagem obtida durante um exame de Fluoroscopia no momento em que é feito o disparo. $\mathrm{O}$ canal 2 do osciloscópio indica o sinal de controle (cor verde) e a onda do canal 1 indica a medição do sinal elétrico captado por uma câmera de ionização (cor amarela), no qual observou-se a energia residual existente.

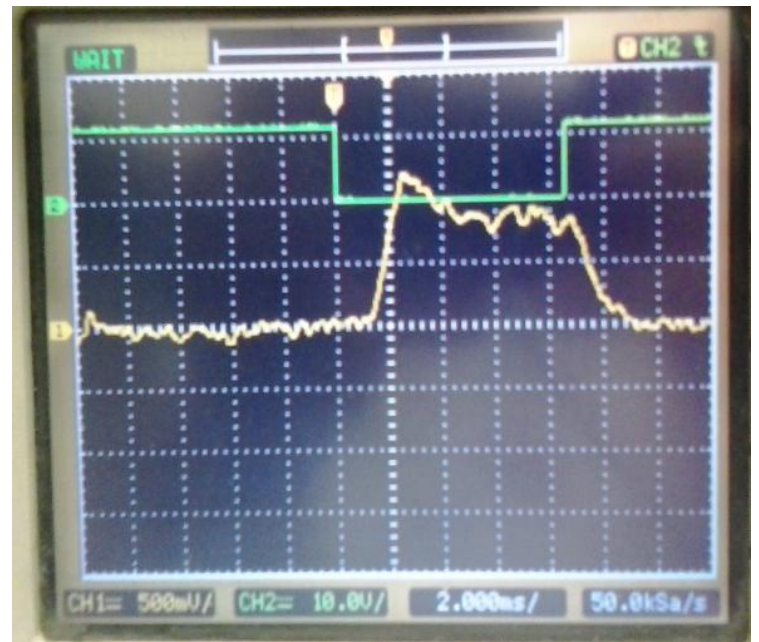

Figura 12 - Osciloscópio utilizado para capturar a forma de onda.

Fonte - Próprio autor.

A partir dessa imagem de curvas de saída, criou-se uma análise dos aspectos construtivos para o tubo de raios- $\mathrm{X}$. 


\section{Analisando a Curva Característica}

Avaliando que os cálculos para esse tipo de circuito envolveriam uma maior complexidade e necessitariam de análises sobre circuitos que reproduzem uma forma de onda em sua saída igual a de um tubo de raios- $X$, algo que na maioria das vezes não está disponível, ou ainda, é considerado de propriedade industrial, desenvolve-se, tendo como base nos parâmetros encontrados, um circuito equivalente no qual obter-se na saída uma onda mais próxima da forma de onda real.

Conforme a Figura 13, o circuito montado a partir de componentes passivos cuja reprodução era uma onda o mais próximo da imagem original. 0 mesmo projetado e simulado no software Proteus.

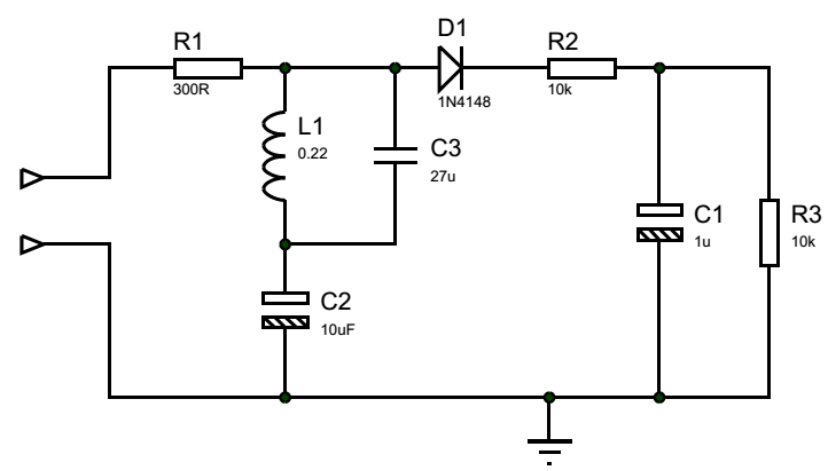

Figura 13 - Circuito elétrico equivalente ao tubo de raios- $X$

Fonte - Próprio autor.

Por meio de componentes utilizados, possibilitou a reprodução de uma forma de onda semelhante à saída no tubo de raios-X. A Figura 14 mostra a saída do circuito através da simulação de um osciloscópio virtual.

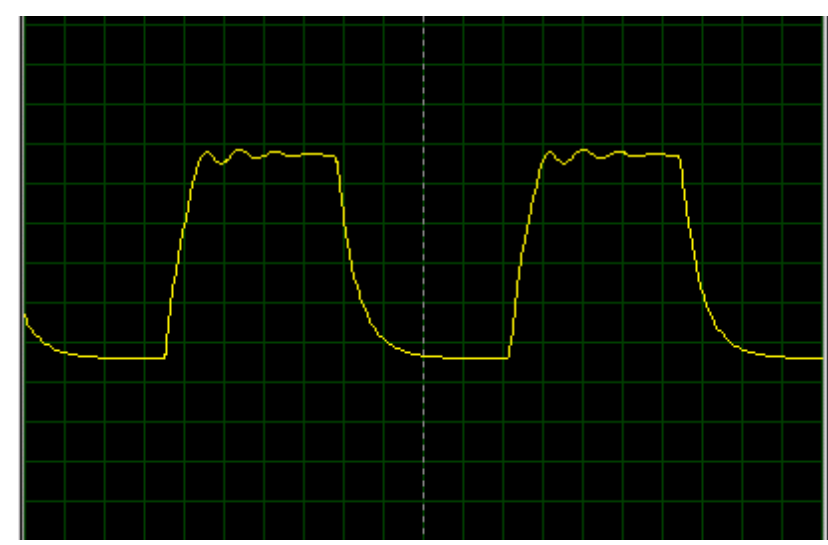

Figura 14 - Forma de onda na saída do circuito simulado

Fonte - Próprio autor.

Depois de criar um circuito equivalente ao tubo de raios- $X$, verificou-se que a forma de onda gerada se assemelhava com a original.

Observando os aspectos construtivos do tubo de raios-X, conforme a Figura 15 é possível verificar que - mesmo possui parâmetros próximos aos componentes utilizados no circuito simulado.

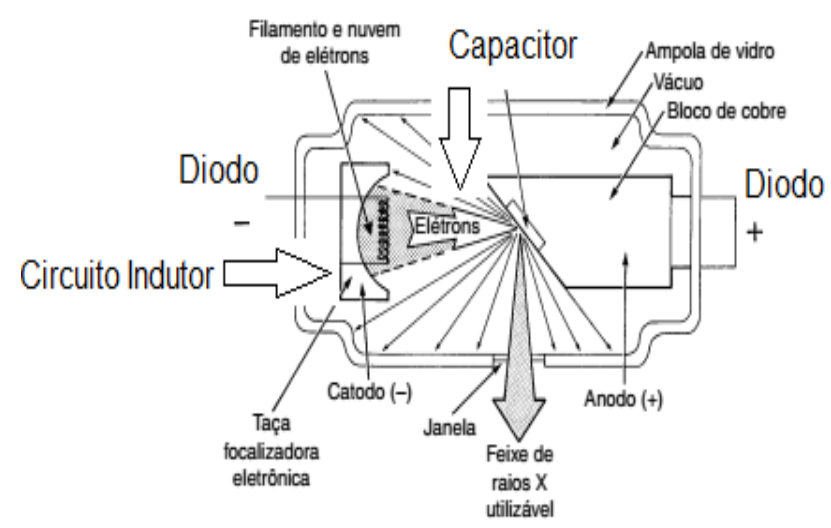

Figura 15 - Estrutura do tubo de raios- $X$ Fonte - WHITE; PHAROAH, 2004, p. 07.

Com a análise da Figura 15 e do circuito simulado criou-se uma metodologia referente ao circuito elétrico equivalente do tubo de raios- $X$ para a curva de tensão da saída.

Do ponto de vista construtivo, identificou-se que o tubo de raios- $X$ possui semelhanças físicas a um capacitor 
de placas paralelas interligadas, em que internamente o mesmo possui vácuo e as placas são submetidas a uma diferença de potencial.

Aliado a isso, o tubo de raios- $X$ se assemelha a um diodo que apresentava um anodo girante e um catodo que confere ao tubo um caráter unidirecional da corrente elétrica.

Em relação à condução da corrente elétrica e sua respectiva variação no tempo, pode ser associado ao tubo de raios-X um caráter indutivo devido aos aspectos construtivos do filamento de tungstênio que, quando submetido a uma variação de corrente elétrica, liberam elétrons em seu interior.

Mediante a análise do gráfico da Figura 13, que mostra a tensão de saída, foram identificados três estados para o comportamento da onda de saída. No primeiro estado a análise da variação das constantes de tempo, observou o comportamento elétrico do tubo de raios- $X$, no qual o circuito se assemelhava a um circuito RC durante a curva de carga do capacitor na qual pode ser demonstrada pela fórmula de um circuito $\mathrm{RC}$ e determinado pela Equação 3.

$$
V_{c}=V\left(1-e^{-t / R C}\right)
$$

Na qual:

$T$ é a constante de tempo

$R$ é a resistência

\section{$C$ é a capacitância}

$V c$ e a tensão de carga

No instante em que não há tensão, o capacitor está descarregado e o circuito se encontra aberto, com 0 aumento da tensão no tempo, a forma de onda é demonstrada na Figura 16.

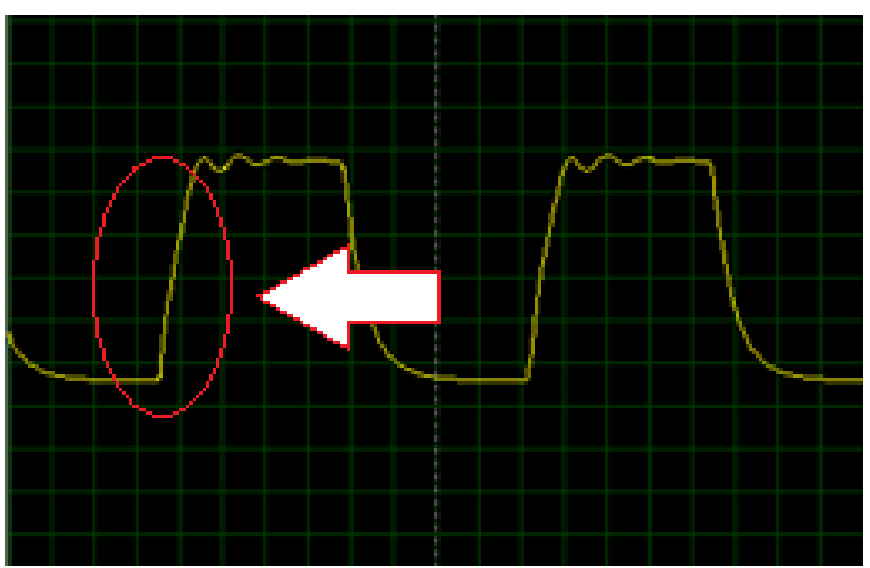

Figura 16 - Forma de onda do circuito RC durante o tempo de carga do capacitor Fonte - Próprio autor.

No segundo momento, a forma de onda entra em um caráter de operação em regime constante não identificando variações bruscas neste estado, "momento caracterizado pelo tempo de disparo". É nesse intervalo que ocorre a irradiação dos tecidos humanos pelos fótons ao atravessarem o corpo e sensibilizarem o detector de imagem, que em seguida é mostrado em um monitor de fluoroscopia.

No terceiro instante de tempo, observou-se uma curva que se assemelha a uma descarga de um circuito RC, desta forma foram identificados constantes aproximadas que descrevem como a tensão varia ao longo do tempo. A Equação 4 se denota a descarga de um circuito RC:

$$
V_{c}=V_{0} \cdot e^{-t / R C}
$$

Em que:

$T$ é a constante de tempo

$R$ é a resistência

$C$ é a capacitância

$V c$ e a tensão de descarga 
$\mathrm{Na}$ qual se verifica que, quando o valor da de carga do capacitor cai, o valor da tensão vai para zero. A Figura 17 demonstra a curva de descarga do circuito RC.

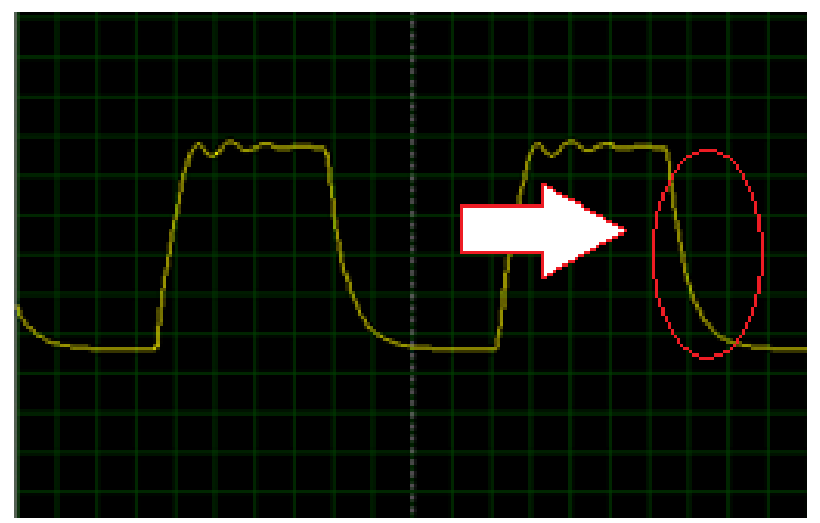

Figura 17 - Forma de onda do circuito RC durante 0 tempo de descarga do capacitor

Fonte - Próprio autor.

A partir dessa etapa foram iniciadas simulações de um circuito capaz de obter uma onda que acarretaria uma menor exposição ao paciente.

\subsection{Simulação e Resultados Obtidos}

Em princípio, o circuito simulado apresentava uma onda de descarga não linear que ser for comparado a um exame real, ocorrera a uma exposição do paciente a uma quantidade de fótons desnecessária, além de não gerar imagem útil para ser processada pelo computador.

Com esse argumento, foi criado um circuito que permitisse reduzir esse tempo de descarga gerado pelo equipamento. Desenvolveu-se uma configuração para o circuito oscilador que foi a base para o disparo demonstrado na Figura 18, criado a partir do software de simulação.

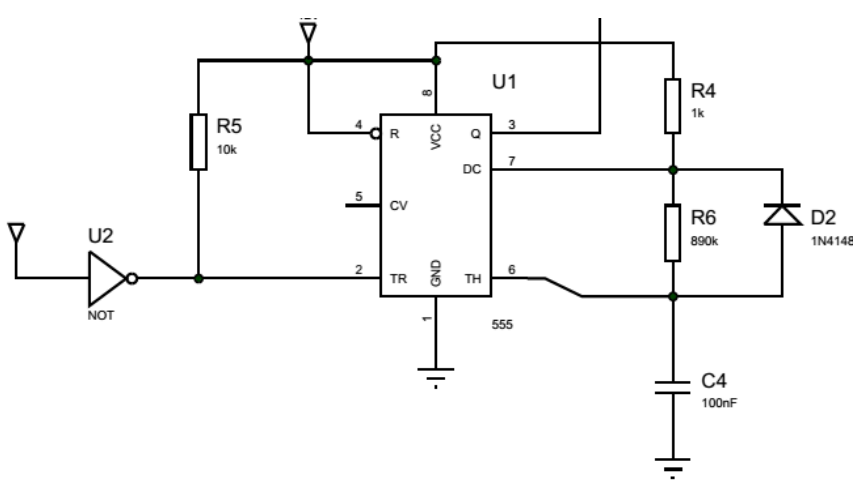

Figura 18 - Circuito de disparo Fonte - Próprio autor.

Os valores do resistor R6 e do capacitor C4 foram estabelecidos para obter a frequência de saída ideal para o circuito de disparo, determinando assim o tempo de condução da onda. A Figura 19 demonstra o circuito de chaveamento e o Snubber.

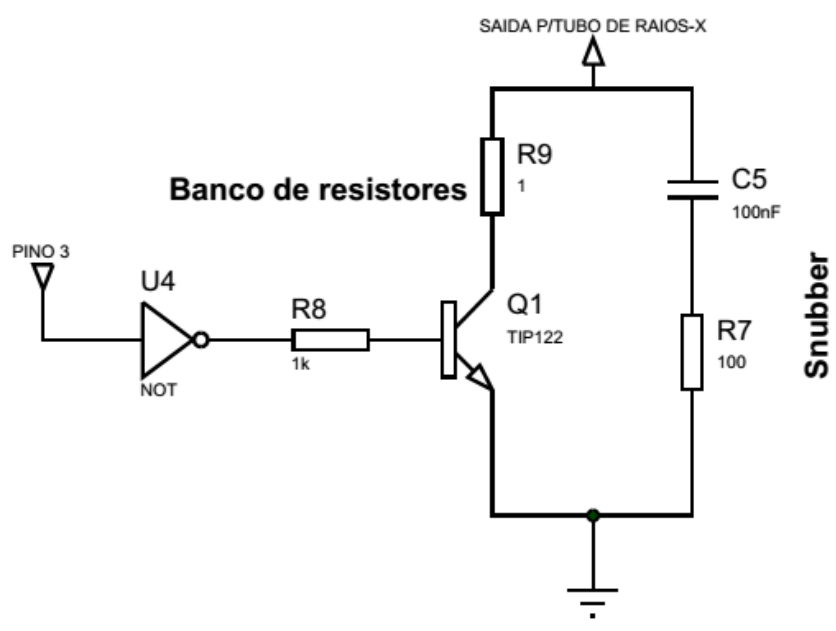

Figura 19 - Circuito driver e circuito Snubber Fonte - Próprio autor.

O circuito é acionado através do sinal que provém do disparo do circuito oscilador, que chega à base do transistor, que por sua vez tem a função de uma chave ora conduzindo e fechando o circuito e ora aberto, fazendo com que o circuito não tenha passagem de corrente. O circuito Snubber, inserido como proteção, tem a função de eliminar a variação de corrente ao longo do tempo em cima do transistor, que o faria queimar, já que a frequência de chaveamento do circuito é na ordem de $\mathrm{kHz}$. E um 
banco de resistores que tem o objetivo de dissipar a tensão residual gerada ao final do disparo.

Ao final da simulação o resultado observado foi que o circuito RC permitiu recriar uma forma de onda equivalente a obtida durante um exame, desta forma o tempo de descarga teria uma configuração não linear na descida da onda. Com a inserção do circuito de descarga, a simulação obteve o seu resultado desejado, de forma a atenuar a onda de descida que não é aproveitada durante o exame, resultado obtido através de um software de simulação. Concluiu-se como o esperado levando em conta que o estudo realizado não representa um circuito real com as particularidades e características de cada componente.

A Figura 20 representa a forma de onda simulada após a inserção do circuito de disparo, na qual são observadas uma redução da curva exponencial e a diminuição da energia desnecessária para o exame e prejudicial ao paciente, na qual a forma de onda de descarga foi atenuada após a inserção do circuito.

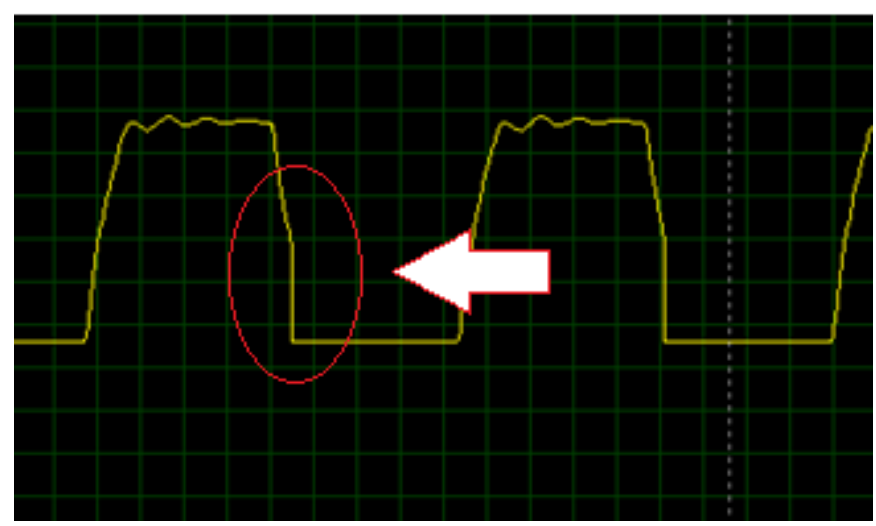

Figura 20 - Forma de onda depois do circuito descarregador montado Fonte - Próprio autor.

\section{ConCLUSÃo}

Conclui-se com este artigo, de forma leviana, o funcionamento de um gerador de alta tensão utilizado em exames de fluoroscopia bem como as características do tubo de raios- $X$, no qual verificou-se que o equipamento emite uma faixa de fótons mesmo depois do final do disparo. Com esse argumento, foi criado e simulado, por meio de um software de simulação, um circuito que foi capaz de diminuir a exposição causada ao paciente no momento da emissão dos raios- $X$ durante 0 exame que não gera nenhum tipo de imagem que possa ser aproveitada.

Demonstrou-se com as simulações a capacidade do circuito em atenuar a descida que antes era semelhante a uma forma de onda exponencial, tornando-o mais eficaz e menos prejudicial, levando em conta que os resultados obtidos se deram por meio de uma simulação, uma demonstração real do circuito envolveria cálculos mais precisos e uma abordagem mais criteriosa para sua criação. 


\section{REFERÊNCIAS}

CORRÊA, M. V; AGUIRRE, L. Identificação não-linear caixa-cinza: Uma revisão e novos resultados. Revista Controle \& Automação, 2004. v. 15, n. 2, p. 18.

DUFFIN, J.; HAYTER, C. R. Baring the sole. The rise and fall of the shoe-fitting fluoroscope. Isis, 2000. v. 91 , n. 2, p. 260-282.

FLÔR, R. D. C.; KIRCHHOF, A. L. C. Uma prática educativa de sensibilização quanto à exposição $a$ radiação ionizante com profissionais de saúde.

Revista Brasileira de Enfermagem, 2006. v. 59, n. 3, p. 274-278.

LINTON, O. W. Medical Applications of X Rays. Beam Line, 1995. p. 25-34.

MADELON, A. F. Z.; SILVA, T. A. Da. Requisitos de proteção radiológica em clínicas odontológicas.

[S.I.]: Instituto de Pesquisas Energéticas e Nucleares, 2015. ISBN 9788578110796.

MINISTERIO DA SAÚDE. Portaria 453, de 01 de junho de 1998. Dispoês sobre as Diretrizes de Proteção Radiológica em Radiodiagnostico Médico e odontólogico.

MIRANDA, J. A. D. E. Desenvolvimento de uma metodologia para a calibração de instrumentos de medida utilizados no controle de qualidade em radiodiagnóstico intervencional Autarquia associada à Universidade de São Paulo Desenvolvimento de uma metodologia para a calibração de i. [S.I.]: INSTITUTO DE PESQUISAS ENERGÉTICAS E NUCLEARES, 2009.
PARIZOTI, A. Otimização de Imagens e Proteção Radiológica em Fluoroscopia. [S.I.]: Universidade de São Paulo, 2008.

SILVA, A. J. Da. Avaliação da dose ocupacional oriunda dos procedimentos especiais guiados por fluoroscopia : cateterismo cardíaco. [S.I.]: Universidade de São Paulo, 2011.

VAREX IMAGING. Special Procedures Tubes. [S.I.], 2017. Disponível em:

$<$ https://www.vareximaging.com/products/oem-specialprocedures $>$.

WHITE, S. C.; PHAROAH, M. J. Radiologia Oral. $5^{\circ}$ ed. Rio de Janeiro: Elsevier Editora Ltda, 2004.

WOMENS HEALTH ADVICE. Coronary Angiography. Kay Collins, 2013. Disponível em: $<$ http://www.womens-health-advice.com/heartdisease/coronary-angiography.html>. Acesso em: 22 maio 2016. 